\title{
Nonlinear finite element analysis of axially crushed cotton fibre composite corrugated tubes
}

\begin{abstract}
It is proven experimentally that introducing corrugation along a shell generator together with a proper advanced composite material will enhance the crashworthiness performance of energy device units. This is because corrugation along the shell generator will force the initial crushing to occur at a predetermined region along the tube generator. On the other hand, a proper composite material offers vast potential for optimally tailoring a design to meet crashworthiness performance requirements. In this paper, the energy absorption characteristics of cotton fibre/propylene corrugated tubes are numerically studied. Finite element simulation using ABAQUS/Explicit was carried out to examine the effects of parametric modifications on the tube's energy absorption capability. Results showed that the tube's energy absorption capability was affected significantly by varying the number of corrugation and aspect ratios. It is found that as the number of corrugations increases, the amount of absorbed energy significantly increases.
\end{abstract}

Keyword: Corrugated tubes, Cotton fibre, Propylene, Non-finite element analysis, ABAQUS 